PROCEEDINGS OF THE

AMERICAN MATHEMATICAL SOCIETY

Volume 126, Number 1, January 1998, Pages 257-267

S $0002-9939(98) 04407-4$

\title{
QUASIPOSITIVE PLUMBING (CONSTRUCTIONS OF QUASIPOSITIVE KNOTS AND LINKS, V)
}

\author{
LEE RUDOLPH
}

(Communicated by Ronald Stern)

Dedicated to Professor Kunio Murasugi

\begin{abstract}
A Seifert surface $S \subset S^{3}=\partial D^{4}$ is a fiber surface if a push-off $S \rightarrow S^{3} \backslash S$ induces a homotopy equivalence; roughly, $S$ is quasipositive if pushing Int $S$ into Int $D^{4} \subset \mathbb{C}^{2}$ produces a piece of complex plane curve. A Murasugi sum (or plumbing) is a way to fit together two Seifert surfaces to build a new one. Gabai proved that a Murasugi sum is a fiber surface iff both its summands are; we prove the analogue for quasipositive Seifert surfaces.

The slice (or Murasugi) genus $g_{s}(L)$ of a link $L \subset S^{3}$ is the least genus of a smooth surface $S \subset D^{4}$ bounded by $L$. By the local Thom Conjecture, $g_{s}(\partial S)=g(S)$ if $S \subset S^{3}$ is quasipositive; we derive a lower bound for $g_{s}(\partial S)$ for any Seifert surface $S$, in terms of quasipositive subsurfaces of $S$.
\end{abstract}

\section{INTRODUCTION}

Murasugi [7], studying certain alternating links (retrospectively, those that fiber), introduced a construction of Seifert surfaces from simpler pieces (namely, fiber surfaces of 2-strand torus links $O\{2, k\})$. Stallings [17], as one of his "constructions of fibered knots and links" (actually, of fiber surfaces) generalized [7] and named the construction "plumbing". Gabai [3] renamed it "Murasugi sum" (reserving "plumbing" for a special case, rather different from that in [7], which had been investigated earlier, cf. [17], [2]), and put the operation in a broader geometric context (leastgenus surfaces and "Reebless foliations"). As applied to fiber surfaces, one aspect of Gabai's slogan [5] that "Murasugi sum is a natural geometric operation" is his theorem that a Murasugi sum is a fiber surface iff the summands are fiber surfaces; in $\S 4$, I use braided Seifert surfaces to prove an analogue.

Theorem. A Murasugi sum is quasipositive iff the summands are quasipositive.

Murasugi [8] was also an early investigator of that "numerical invariant of link types" now called "slice genus" or "Murasugi genus". A theorem of Kronheimer and Mrowka [6] implies that, if $S$ is a quasipositive Seifert surface, then $g_{s}(\partial S)=g(S)$; this implication was shown in [14], where it was used to derive a lower bound (the "slice-Bennequin inequality") for the slice genus of any link presented as a closed braid. In $\S 5$, I derive a more general (and often stronger) lower bound for $g_{s}(L)$ in

Received by the editors October 1, 1995.

1991 Mathematics Subject Classification. Primary 57M25; Secondary 32S55, 14H99.

Key words and phrases. Murasugi sum, plumbing, quasipositive, slice genus, Thom conjecture.

Partially supported by grants from CAICYT, NSF (DMS-8801915, DMS-9504832), and CNRS. 
terms of any Seifert surface $S$ with $L=\partial S$. As an application, I combine this with the Theorem to estimate the slice genus of certain plumbed links. For instance, let $S$ be a Seifert surface plumbed from unknotted, twisted annuli according to a weighted tree each vertex of which has even (total) weight; many arborescent links (including many of the fibered ones, cf. [4]) have such Seifert surfaces.

Proposition. Let $\partial S$ have $r$ components. If there are $p$ vertices with positive weight and $q$ with negative weight, then $g_{s}(\partial S) \geq(1-r+|p-q|) / 2$.

Notation and general definitions are established in $\S 2$. A braid-free exposition of braided Seifert surfaces and quasipositivity is given in $\S 3$.

\section{Preliminary notations and Definitions}

The notations $A:=B$ and $B=: A$ both define $A$ to mean $B$. For any cartesian product (e.g., $\mathbb{R}^{3}$ ), $\operatorname{pr}_{i}$ denotes projection on the $i$ th factor; similarly for $\operatorname{pr}_{i, j}$.

2.1. Sets. Write two $(Y)$ for the set of 2-element subsets of $Y$. If $Y$ is (totally) ordered, such notations as $\{s, t\} \in \operatorname{two}(Y),\{i, j\}: C \rightarrow \operatorname{two}(Y)$, etc., typically presuppose that $s<t, i(c)<j(c)$ for all $c \in C$, etc. If $\{s, t\} \in \operatorname{two}(Y)$ and there is no $u \in Y$ such that $s<u<t$, then $t$ is the successor of $s$ in $Y$. If $\{s, t\},\left\{s^{\prime}, t^{\prime}\right\} \in$ two $(Y),\{s, t\} \neq\left\{s^{\prime}, t^{\prime}\right\}$, then $\{s, t\}$ and $\left\{s^{\prime}, t^{\prime}\right\}$ link iff either $s<s^{\prime}<t<t^{\prime}$ or $s^{\prime}<s<t^{\prime}<t$, unlink iff either $s<s^{\prime}<t^{\prime}<t$ or $s^{\prime}<s<t<t^{\prime}$, and touch at $u$ iff $\{s, t\} \cap\left\{s^{\prime}, t^{\prime}\right\}=\{u\}$. Write $\mathbf{n}:=\{1, \ldots, n\}$ for $n \in \mathbb{N}:=\{1,2, \ldots\}$, and $\mathbf{0}:=\varnothing$. If $\operatorname{card}(Y)=: m<\infty$, then $\#_{Y}: \mathbf{m} \rightarrow Y$ denotes the increasing bijection; so, e.g., $\min Y=\#_{Y}(1), \max Y=\#_{Y}(m)$. (Read $\#_{Y}$ as "count $Y$ ".)

2.2. Manifolds. Spaces, maps, etc., are piecewise smooth. Manifolds may have boundary and are always oriented; in particular, $\mathbb{R}, \mathbb{C}^{n}$, and $S^{2 n-1} \subset \mathbb{C}^{n}$ have standard orientations, as does $\mathbb{R}^{3}$ which is identified with the complement of a point $\infty \in S^{3}$. If $M$ is a manifold, then $-M$ denotes $M$ with its orientation reversed (and, where notation requires it, $+M$ denotes $M$ ). A submanifold $P \subset M$ is proper (resp., interior; boundary) if $\partial P=P \cap \partial M$ (resp., $P \subset \operatorname{Int} M ; P \subset \partial M$ ). For a suitable subset $Q \subset M, N_{M}(Q)$ denotes a closed regular neighborhood of $Q$ in $(M, \partial M)$. For a suitable codimension-1 submanifold $Q \subset M$, a collaring of $Q$ in $M$ is an orientation-preserving embedding $\operatorname{col}_{Q \subset M}: Q \times[0,1] \rightarrow M$ such that $\operatorname{col}_{Q \subset M}(q, 0)=q$ for all $q$; a collar is the image of a collaring. The pushoff of $Q$ is the embedding $Q \rightarrow M \backslash Q: q \mapsto \operatorname{col}_{Q \subset M}(q, 1)$; let $Q^{+}$denote the image of $Q$ by the push-off, oriented so that the push-off preserves orientation (thus $Q^{+} \subset \partial\left(\operatorname{col}_{Q \subset M}(Q \times[0,1])\right)$ has the conventional, "outward normal" orientation, whereas the inclusion $Q \subset \partial\left(\operatorname{col}_{Q \subset M}(Q \times[0,1])\right)$ reverses orientation).

An arc is a manifold diffeomorphic to $[0,1]$. A surface is a compact 2-manifold no component of which has empty boundary. A handle decomposition

$$
S=\bigcup_{x \in X} h_{x}^{(0)} \cup \bigcup_{z \in Z} h_{z}^{(1)}
$$

of a surface $S$ is not necessarily ordered; it is understood that if $z_{1} \neq z_{2}$ then the attaching regions $h_{z_{t}}^{(1)} \cap \partial\left(\bigcup_{x \in X} h_{x}^{(0)}\right)(t=1,2)$ are disjoint. Write $S^{(0)}:=$ $\bigcup_{x \in X} h_{x}^{(0)}, S^{(1)}:=\bigcup_{z \in Z} h_{z}^{(1)}$ : thus, $S^{(0)} \cap S^{(1)}$ is the union of the attaching regions. For $p \in S^{(i)}$ (resp., $P \subset S^{(i)}$ ), write $h^{(i)}(p)$ (resp., $h^{(i)}(P)$ ) for the unique $i$-handle of (2.2.1) containing $p$ (resp., $P$ ). 
A core (resp., transverse) arc of a 1-handle $h^{(1)}$ is any proper arc which joins interior points of the two components of the attaching region of $h^{(1)}$ (resp., the complement in $\partial h^{(1)}$ of the attaching region of $h^{(1)}$ ). Note that, if $S$ is a surface and $\alpha \subset S$ is an arc, then $S$ has some handle decomposition (2.2.1) such that $\alpha$ is a core arc (resp., a transverse arc) of some $h_{z}^{(1)}, z \in Z$, iff $\alpha$ is interior (resp., proper).

2.3. Stars and patches. An arc $\tau$ contained in a surface $S$ is half-proper if one endpoint of $\tau$ belongs to $\partial S$ and the rest of $\tau$ is contained in $\operatorname{Int} S$. An $n$-star $\psi \subset S$ is a union of $n$ half-proper arcs, the rays of $\psi$, which are pairwise disjoint except for a common endpoint in Int $S$, the center of $\psi$; the tip of a ray $\tau \subset \psi$ is that endpoint $\operatorname{tip}(\tau)$ of $\tau$ which is not the center of $\psi$. An n-patch is the regular neighborhood $N_{S}(\psi)$ of an $n$-star. An $n$-patch is a 2-disk naturally endowed with the structure of a $2 n$-gon of which the edges are alternately boundary arcs and proper $\operatorname{arcs}$ in $S$.

An $n$-star $\psi \subset S$ is transverse to the handle decomposition (2.2.1) of $S$ if

(2.3.1) the center of $\psi$ lies in $\operatorname{Int} S^{(0)}$,

(2.3.2) for each ray $\tau \subset \psi, \operatorname{tip}(\tau) \in \partial S^{(0)} \backslash S^{(1)}$, and

(2.3.3) each ray $\tau \subset \psi$ is transverse to $S^{(0)} \cap S^{(1)}$.

Let $\psi$ be transverse to (2.2.1). Define $c(\psi):=\operatorname{card}\left(\psi \cap S^{(0)} \cap S^{(1)}\right)$. A ray $\tau \subset \psi$ will be called long if $\tau \not \subset S^{(0)}$; so $c(\psi)=0$ iff no ray $\tau \subset \psi$ is long iff $\psi \subset S^{(0)}$ iff $\psi \subset h_{x}^{(0)}$ for some $x \in X$. Let $\tau \subset \psi$ be a long ray. The tail of $\tau$, denoted tail $(\tau)$, is that component arc of $\tau \cap S^{(0)}$ which has $\operatorname{tip}(\tau)$ as one endpoint; the coccyx of $\tau$, denoted $\operatorname{coccyx}(\tau)$, is the other endpoint of tail $(\tau)$. Call $\tau$ loose if either of the two 2-disks into which tail $(\tau)$ divides $h^{(0)}(\operatorname{tail}(\tau))$ has empty intersection with $S^{(1)} \backslash h^{(1)}(\operatorname{coccyx}(\tau))$. Call $\tau$ slack if $\tau$ contains an arc $\alpha$ with both endpoints on the same component of $S^{(0)} \cap S^{(1)}$. Call $\psi$ minimal with respect to (2.2.1) if $c(\psi) \leq c\left(\psi^{\prime}\right)$ for every $n$-star $\psi^{\prime} \subset S$ which is transverse to (2.2.1) and ambient isotopic to $\psi$ on $S$. The following is easily proved.

2.3.4. Lemma. If $\psi$ is minimal, then no ray of $\psi$ is either slack or loose.

2.4. Seifert surfaces. A Seifert surface is a surface $S \subset S^{3}$. A link $L$ is the boundary of a Seifert surface; a knot is a connected link. If $K$ is a knot, then $A(K, n)$ denotes any Seifert surface diffeomorphic to an annulus such that $K \subset \partial A(K, n)$ and the linking number in $S^{3}$ of $K$ and $K^{\prime}:=\partial A(K, n) \backslash K$ is $-n$ (that is, the Seifert matrix $\theta_{A(K, n)}$ is $\left.[n]\right)$. Since clearly $K^{\prime}$ and $-K$ are ambient isotopic, so are $A(K, n)$ and $A(-K, n) ; A(K, n)$ and $-A(K, n)$ are also ambient isotopic.

A Seifert surface $S$ is: (1) a fiber surface (and $\partial S$ is a fibered link) if there exists a fibration $\varphi: S^{3} \backslash \partial S \rightarrow S^{1}$ such that Int $S$ is a fiber of $\varphi$ and the closure of every fiber of $\varphi$ is a Seifert surface with boundary $\partial S$; (2) least-genus if $S$ maximizes Euler characteristic among all Seifert surfaces with boundary $\partial S$; (3) incompressible if, whenever $D^{2} \subset S^{3}$ is a disk such that $D^{2} \cap S=\partial D^{2}$, then $\partial D^{2}$ bounds a disk on $S$. The following facts are well known (cf., e.g., [17], [3]-[5]): (1) $S$ is a fiber surface iff $S$ is connected and a push-off induces an isomorphism $\pi_{1}(S) \rightarrow \pi_{1}\left(S^{3} \backslash S\right)$;

(2) a least-genus surface $S$ is incompressible; (3) a fiber surface is least-genus, and up to ambient isotopy it is the unique incompressible surface with its boundary; (4) $A(K, n)$ is least-genus iff $(K, n) \neq(O, 0) ;(5) A(K, n)$ is a fiber surface iff $(K, n)=(O,-1)$ or $(K, n)=(O, 1)$. The annulus $A(O,-1)$ (resp., $A(O, 1))$ is called a positive (resp., negative) Hopf annulus (sometimes "Hopf band"); the sign convention reflects the linking number of the components of $\partial A(O, \mp 1)$. 
2.5. Stallings plumbing; Murasugi sum. Let $S$ be a Seifert surface. Let $B \subset S^{3}$ be a 3 -ball. Let $S_{1}:=S \backslash \operatorname{Int} B, S_{2}:=S \cap B, N:=S_{1} \cap S_{2}=S \cap \partial B$. Say that $B$ deplumbs $S$ into plumbands $S_{1}$ and $S_{2}$ if $S_{1}$ is a Seifert surface and $N \subset S_{1}$ is an $n_{1}$-patch (for some $n_{1}$ ); in this case, necessarily $S_{2}$ is also a Seifert surface, and $N \subset S_{2}$ is an $n_{2}$-patch, where, it should be noted, $n_{2}$ need not equal $n_{1}$.

If $S_{1}$ and $S_{2}$ are Seifert surfaces, $N_{s} \subset S_{s}$ is an $n_{s}$-patch, and $h: N_{1} \rightarrow N_{2}$ is an orientation-preserving diffeomorphism with

$$
h\left(N_{1} \cap \partial S_{1}\right) \cup\left(N_{2} \cap \partial S_{2}\right)=\partial N_{2}
$$

then $S_{2}$ is ambient isotopic to $S_{2}^{\prime}$ such that $S:=S_{1} \cup S_{2}^{\prime}$ is a Seifert surface deplumbed by a 3-ball $\operatorname{col}_{S_{1} \subset S^{3}}\left(N_{1}\right)$ into plumbands $S_{1}$ and $S_{2}^{\prime}$, and the isotopy carries $h$ to the identity $N_{1} \rightarrow N_{2}^{\prime}$. Call $S$ a Stallings plumbing of $S_{1}$ and $S_{2}$ along $h$ (cf. [17]) and denote it by $S_{1} *_{h} S_{2}$, or just $S_{1} * S_{2}$ when it is safe to leave $h$ inexplicit. (It is possible, and in a sense typical, that changing the isotopy class of $h$ will change the ambient isotopy type of $S_{1} *_{h} S_{2}$.) There are $n_{s}$-stars $\psi_{1} \subset S_{1}$ and $\psi_{2}^{\prime} \subset S_{2}^{\prime}$ (corresponding to $\psi_{2} \subset S_{2}$ ) such that $\psi_{1} \cup \psi_{2}^{\prime}$ is an $\left(n_{1}+n_{2}\right)$-star on the disk $N_{1}=N_{2}^{\prime}$; the combinatorics of the interleaving of the rays of $\psi_{1}$ and $\psi_{2}^{\prime}$ in $N_{1}=N_{2}^{\prime}$ contains all the information needed to (re)construct $S_{1} *_{h} S_{2}$.

On its face, Stallings plumbing is a strict generalization of Murasugi sum (cf. [7], [3], [4]), its seemingly special case in which $n_{1}=n_{2}$ and (2.5.1) is supplemented by

$$
h\left(N_{1} \cap \partial S_{1}\right) \cap\left(N_{2} \cap \partial S_{2}\right)=\partial\left(N_{2} \cap \partial S_{2}\right) .
$$

In fact, however, it is easy to see that (up to ambient isotopy) every Stallings plumbing is a Murasugi sum of the same plumbands. The distinction is nonetheless useful and will be maintained here.

Stallings [17] showed that any Stallings plumbing of fiber surfaces is a fiber surface. Gabai [3] showed that any Murasugi sum (viz., Stallings plumbing) of least-genus surfaces is least-genus, and, further, that if $S_{1} *_{h} S_{2}$ is a fiber (resp., least-genus) surface, then $S_{1}$ and $S_{2}$ are fiber (resp., least-genus) surfaces.

\section{Braided Seifert Surfaces AND QUASIPOSITIVITY}

3.1. Braided Seifert surfaces. Let $\mathbb{R}_{\geq \xi}$ (resp., $\mathbb{R}_{\leq \xi}$ ) denote $\{t \in \mathbb{R}: t \geq \xi\}$ (resp., $\{t \in \mathbb{R}: t \leq \xi\}$ ). A Seifert surface $S \subset \mathbb{R}^{3}=S^{3} \backslash\{\infty\}$ is braided if it has a handle decomposition (2.2.1) with $X, Z \subset \mathbb{R}$, which satisfies the following conditions:

(3.1.1) for $x \in X, S \cap\{x\} \times \mathbb{R}_{\geq_{0}} \times \mathbb{R}=h_{x}^{(0)}$, and $h_{x}^{(0)}$ induces the same orientation on $\{x\} \times\{0\} \times \mathbb{R}$ as $\operatorname{pr}_{3} \mid\{x\} \times\{0\} \times \mathbb{R}:\{x\} \times\{0\} \times \mathbb{R} \rightarrow \mathbb{R}$

(3.1.2) for $z \in Z, \operatorname{pr}_{3} \mid h_{z}^{(1)}$ is Morse with exactly one (interior) critical point, of index 1 (so $S \cap \mathbb{R} \times \mathbb{R}_{\leq 0} \times\{z\}$ is the union of a core arc and a transverse arc of $h_{z}^{(1)}$ meeting transversely there).

Combinatorial data characterizing $S$ is readily extracted from (3.1.1-2), to wit:

(3.1.3) $\{i, j\}: Z \rightarrow \operatorname{two}(X)$ such that $h_{z}^{(1)}$ is attached to $h_{i(z)}^{(0)}$ and $h_{j(z)}^{(0)}$,

(3.1.4) $\varepsilon: Z \rightarrow\{+,-\}$ such that the positive normal vector to $S$ at the critical point of $\operatorname{pr}_{3} \mid h_{z}^{(1)}$ is a positive multiple of $\varepsilon(t) D \mathrm{pr}_{3}$.

Then $S$ is determined, up to isotopy through braided Seifert surfaces with $X$ and $Z$ fixed, by $(\{i, j\}, \varepsilon)$. Denote any braided Seifert surface with data $(\{i, j\}, \varepsilon)$ by $S[i, j, \varepsilon]$. Call $S$ standardized if $X=\mathbf{n}, Z=\mathbf{k}$. Clearly, $S[i, j, \varepsilon]$ is isotopic (through braided Seifert surfaces) to its standardization $S\left[i^{\prime}, j^{\prime}, \varepsilon^{\prime}\right], i^{\prime}:=\left(\#_{X}\right)^{-1} \circ i \circ \#_{Z}, j^{\prime}:=$ 

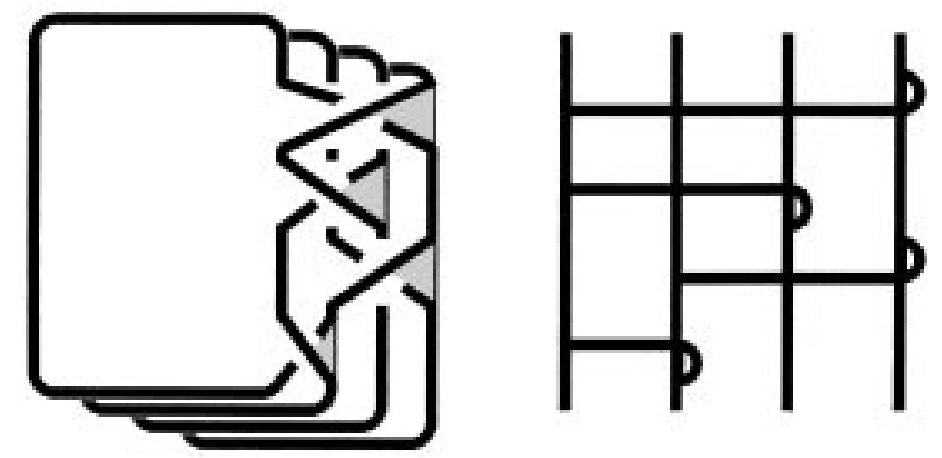

Figure 1. $S[i, j, \varepsilon]$, for $(i, j, \varepsilon): 1 \mapsto(1,2,-), 2 \mapsto(2,4,+), 3 \mapsto$ $(1,3,-), 4 \mapsto(1,4,+)$; the corresponding charged fence diagram.

$\left(\#_{X}\right)^{-1} \circ j \circ \#_{Z}, \varepsilon^{\prime}:=\varepsilon \circ \#_{Z}$. It is, however, convenient not to be limited to standardized braided Seifert surfaces.

Let $S$ be a braided Seifert surface. Then the inverse $-S$ is surely not braided; however, $-S$ is isotopic to the braided surface $-R(S)$, where $R: \mathbb{R}^{3} \rightarrow \mathbb{R}^{3}$ : $(x, y, z) \mapsto(-x, y,-z)$. On the other hand, the mirror image $\operatorname{Mir}(S)$ is braided, where Mir $: \mathbb{R}^{3} \rightarrow \mathbb{R}^{3}:(x, y, z) \mapsto(x, y,-z)$. Let $\rho_{m}: \mathbf{m} \rightarrow \mathbf{m}: i \mapsto m+1-i$. If $S[i, j, \varepsilon]$ is standardized then $-S[i, j, \varepsilon]$ is isotopic to $S\left[\rho_{n} \circ j \circ \rho_{k}, \rho_{n} \circ i \circ \rho_{k}, \varepsilon \circ \rho_{k}\right]$, and $\operatorname{Mir}(S[i, j, \varepsilon])$ is isotopic to $S\left[i \circ \rho_{k}, j \circ \rho_{k},-\varepsilon \circ \rho_{k}\right]$.

Remark on the figures. Perhaps the most convenient graphic portrayal of a braided Seifert surface, one which conveys all its combinatorics at a glance, is a charged fence diagram (cf. [13]). The fence diagram corresponding to $S=S[i, j, \varepsilon]$ is the union (in the $(x, z)$-plane) of the set $\operatorname{pr}_{1,3}\left(S^{(0)}\right)$ of posts and the set $\operatorname{pr}_{1,3}\left(S^{(1)} \cap \mathbb{R} \times \mathbb{R}_{\leq 0} \times Z\right)$ of wires; the corresponding charge, which formally is the map induced by $\varepsilon$ from the set of wires to $\{+,-\}$, is conveniently depicted by adding a "hook" of the correct handedness to the end of each wire. Figure 1 shows a braided Seifert surface and a corresponding charged fence diagram.

3.2. Braided Stallings plumbing. Let $S_{s}:=S\left[i_{s}, j_{s}, \varepsilon_{s}\right](s=1,2)$ be braided Seifert surfaces. If

(3.2.1) $\max X_{2}=\min X_{1}, Z_{1} \cap Z_{2}=\varnothing$

and we set $X=X_{1} \cup X_{2}, Z=Z_{1} \cup Z_{2}$, and define $(\{i, j\}, \varepsilon)$ by $(\{i, j\}, \varepsilon) \mid Z_{s}=$ $\left(\left\{i_{s}, j_{s}\right\}, \varepsilon_{s}\right)$, then $S[i, j, \varepsilon]$ is a Stallings plumbing $S_{1} *_{h} S_{2}$ (deplumbed by the 3-ball $\left.\left(\left\{\min X_{1}\right\} \times \mathbb{R} \times \mathbb{R}\right) \cup\{\infty\} \subset \mathbb{R}^{3} \cup\{\infty\}=S^{3}\right)$ which satisfies a further constraint:

(3.2.2) $N_{1} \subset S_{1}$ (resp., $N_{2} \subset S_{2}$ ) lies in the leftmost (resp., rightmost) 0-handle of the handle decomposition (2.2.1) of $S_{1}$ (resp., $S_{2}$ ).

Call such a Stallings plumbing braided. (The plumbings used by Stallings to prove Theorem 2 of [17] are, inexplicitly, braided.)

Given $S_{1}, S_{2}^{\prime}$, and an increasing injection $H: Z_{2}^{\prime} \rightarrow \mathbb{R} \backslash Z_{1}$, if $S_{2}:=S\left[i_{2}, j_{2}, \varepsilon_{2}\right]$ is a braided Seifert surface with the same standardization as $S_{2}^{\prime}$ such that $X_{2}:=$ $X_{2}^{\prime}+\left(\min X_{1}-\max X_{2}^{\prime}\right), Z_{2}:=H\left(Z_{2}^{\prime}\right)$, then (3.2.1) and (3.2.2) are satisfied. The isotopy class of $h$ for the resulting braided Stallings plumbing $S_{1} *_{h} S_{2}$ is determined by $H$ (actually, by the homotopy class of $\left.H \mid: j_{2}^{\prime-1}\left(\max X_{2}^{\prime}\right) \rightarrow \mathbb{R} \backslash i_{1}^{-1}\left(\min X_{1}\right)\right)$. 




a deflation

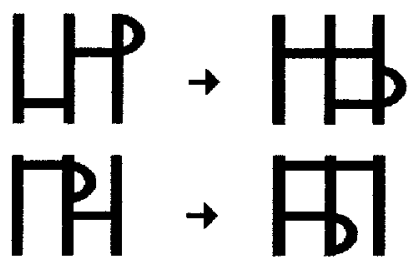

two slides up

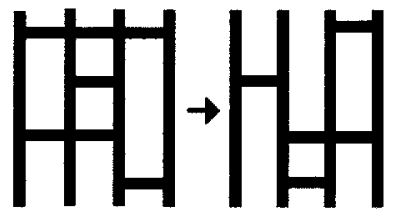

a twirl

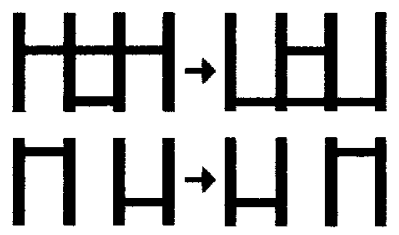

two slips

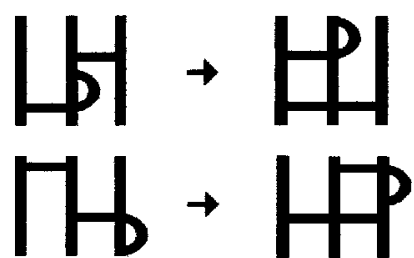

two slides down

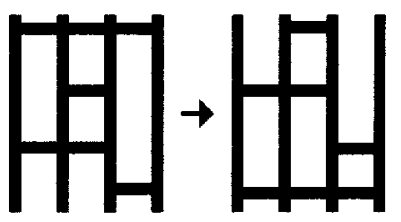

a turn

Figure 2

3.3. Inflations, deflations, slips, slides, twirls, and turns. Each of the following operations replaces a braided Seifert surface $S:=S[i, j, \varepsilon]$ with an ambient isotopic braided Seifert surface $S^{\prime}:=S\left[i^{\prime}, j^{\prime}, \varepsilon^{\prime}\right]$ having a different standardization. The operations are illustrated with fence diagrams in Figure 2.

(3.3.1) Suppose that for some $x_{0} \in X$, there exists a unique $z_{0} \in Z$ with $x_{0} \in$ $\{i, j\}\left(z_{0}\right)$; let $X^{\prime}:=X \backslash\left\{x_{0}\right\}, Z^{\prime}:=Z \backslash\left\{z_{0}\right\},\left(\left\{i^{\prime}, j^{\prime}\right\}, \varepsilon^{\prime}\right)(z):=(\{i, j\}, \varepsilon)(z)$ for $z \in Z^{\prime}$. Say that $S^{\prime}$ is obtained from $S$ by a deflation, and that $S$ is obtained from $S^{\prime}$ by an inflation of $\operatorname{sign} \varepsilon\left(z_{0}\right)$.

(3.3.2) Suppose that $z_{2}$ is the successor of $z_{1}$ in $Z$, and $\{i, j\}\left(z_{1}\right)$ and $\{i, j\}\left(z_{2}\right)$ unlink; let $\left(\left\{i^{\prime}, j^{\prime}\right\}, \varepsilon^{\prime}\right)\left(z_{s}\right):=(\{i, j\}, \varepsilon)\left(z_{3-s}\right)(s=1,2),\left(\left\{i^{\prime}, j^{\prime}\right\}, \varepsilon^{\prime}\right)(z):=(\{i, j\}, \varepsilon)(z)$ for $z \in Z \backslash\left\{z_{1}, z_{2}\right\}$. Say $S^{\prime}$ is obtained from $S$ by a slip.

(3.3.3) Suppose that $z_{2}$ is the successor of $z_{1}$ in $Z$, and $\{i, j\}\left(z_{1}\right)$ and $\{i, j\}\left(z_{2}\right)$ touch at $j\left(z_{s}\right)=i\left(z_{3-s}\right)$. (A) Suppose either $s=1$ and $\varepsilon\left(z_{2}\right)=+$, or $s=$ 2 and $\varepsilon\left(z_{2}\right)=-$; let $\left(\left\{i^{\prime}, j^{\prime}\right\}, \varepsilon^{\prime}\right)\left(z_{1}\right):=(\{i, j\}, \varepsilon)\left(z_{2}\right)$ and $\left(\left\{i^{\prime}, j^{\prime}\right\}, \varepsilon^{\prime}\right)\left(z_{2}\right):=$ $\left(\left\{i\left(z_{s}\right), j\left(z_{3-s}\right)\right\}, \varepsilon\left(z_{1}\right)\right)$. (B) Suppose either $s=1$ and $\varepsilon\left(z_{1}\right)=+$, or $s=2$ and $\varepsilon\left(z_{1}\right)=-$; let $\left(\left\{i^{\prime}, j^{\prime}\right\}, \varepsilon^{\prime}\right)\left(z_{1}\right):=\left(\left\{i\left(z_{s}\right), j\left(z_{3-s}\right)\right\}, \varepsilon\left(z_{2}\right)\right)$ and $\left(\left\{i^{\prime}, j^{\prime}\right\}, \varepsilon^{\prime}\right)\left(z_{2}\right):=$ $(\{i, j\}, \varepsilon)\left(z_{1}\right)$. In each case, let $\left(\left\{i^{\prime}, j^{\prime}\right\}, \varepsilon^{\prime}\right)(z):=(\{i, j\}, \varepsilon)(z)$ for $z \in Z \backslash\left\{z_{1}, z_{2}\right\}$. Say $S^{\prime}$ is obtained from $S$ by a slide up (resp., slide down) in cases (A) (resp., (B)).

(3.3.4) Let $x^{\prime}>\max X$ and $X^{\prime}:=X \backslash\{\min X\} \cup\left\{x^{\prime}\right\}$; let $f: X \rightarrow X^{\prime}$ be defined by $f \mid\left(X \cap X^{\prime}\right)=\operatorname{id}_{X \cap X^{\prime}}, f(\min X)=x^{\prime}$. Let $\left(\left\{i^{\prime}, j^{\prime}\right\}, \varepsilon^{\prime}\right)=(\{f \circ i, f \circ j\}, \varepsilon)$. Say $S^{\prime}$ is obtained from $S$ by a twirl.

(3.3.5) Let $z^{\prime}>\max Z, Z^{\prime}:=Z \backslash\{\min Z\} \cup\left\{z^{\prime}\right\}$; let $g: Z^{\prime} \rightarrow Z$ be defined by $g \mid\left(Z \cap Z^{\prime}\right)=\operatorname{id}_{Z \cap Z^{\prime}}, g\left(z^{\prime}\right)=\min Z$. Let $\left(\left\{i^{\prime}, j^{\prime}\right\}, \varepsilon^{\prime}\right)=(\{i, j\}, \varepsilon) \circ g$. Say $S^{\prime}$ is obtained from $S$ by a turn. 


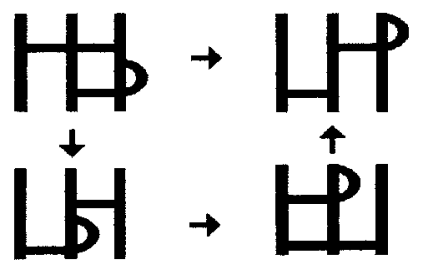

a bent slide

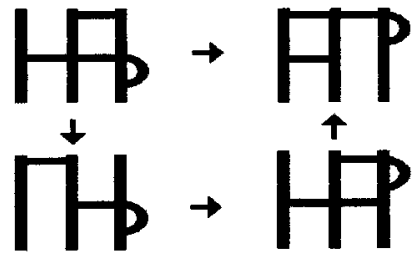

a doubly-bent slide

Figure 3

Remark. Other operations preserving isotopy type can be obtained by composing slides, twirls, and turns: the inverse (up to standardization) of a twirl (resp., turn) is the composition of twirls (resp., turns); the conjugate of a slide by twirls is either a bent slide (the inverse of a slide) or a doubly-bent slide, cf. Figure 3. Question: if $S[i, j, \varepsilon]$ and $S\left[i^{\prime}, j^{\prime}, \varepsilon^{\prime}\right]$ are isotopic (as Seifert surfaces), then do they differ (up to standardization) only by inflations, deflations, slips, slides, twirls, and turns?

3.4. Quasipositivity. A Seifert surface $S \subset S^{3}$ is quasipositive if it is ambient isotopic to some $S[i, j,+]$ (i.e., $\varepsilon$ is the constant function + ). Note that $S$ is quasipositive iff $-S$ is.

Every Seifert surface is ambient isotopic to a braided Seifert surface [9], but not every Seifert surface is quasipositive [11]. A quasipositive Seifert surface is incompressible [10]. In fact, something much stronger is true: if $S$ is quasipositive, then the proper surface $S^{\prime}=\operatorname{col}_{S^{3} \subset D^{4}}(\partial S \times[0,1] \cup S \times\{1\}) \subset D^{4}$ obtained by pushing Int $S$ into Int $D^{4}$ is ambient isotopic to $D^{4} \cap \Gamma$ for some smooth complex algebraic curve $\Gamma:=f^{-1}(0), f(z, w) \in \mathbb{C}[z, w]$, cf. [9], [10] (by no means do all such "pieces of complex plane curve" so arise, but many interesting examples, e.g., the Milnor fiber of a singularity, do), and it follows [14] from work of Kronheimer and Mrowka [6] that $S$ maximizes Euler characteristic among all surfaces smoothly embedded in $D^{4}$ sharing its boundary — in particular, $S$ is least-genus.

3.5. Characterization of quasipositive Seifert surfaces. A subset $W$ of a surface $F$ is full if no component of $F \backslash W$ is contractible.

3.5.1. Theorem [12]. A Seifert surface $S$ is quasipositive iff, for some $n>0, S$ is ambient isotopic to a full subsurface of a fiber surface of the torus link $O\{n, n\}$, i.e., the link of the singularity at the origin of the complex plane curve $z^{n}+w^{n}=0$.

3.5.2. Corollary [12]. A full subsurface of a quasipositive Seifert surface is quasipositive.

\section{Murasugi SUm PRESERVES QUASIPOSITIVITY}

4.1. Stars on braided Seifert surfaces. Let $S:=S[i, j, \varepsilon]$ be a braided Seifert surface with handle decomposition (2.2.1) satisfying 3.1.1-4. Define an $n$-star $\psi \subset S$ to be braided if it satisfies

(4.1.1) the center of $\psi$ is an interior point of $S^{(0)}$,

(4.1.2) for each ray $\tau \subset \psi, \operatorname{tip}(\tau) \subset X \times\{0\} \times \mathbb{R}$,

(4.1.3) $\psi$ is transverse (as in 2.3) to (2.2.1). 
Any $n$-star on $S$ is isotopic to a braided $n$-star which is minimal (as in 2.3). Let $\psi$ be minimal and braided. Let the ray $\tau \subset \psi$ be long. Then $\operatorname{tip}(\tau)$ and $\operatorname{coccyx}(\tau)$ are the endpoints of a line segment on $\partial h^{(0)}(\operatorname{tail}(\tau)) \cap \mathbb{R} \times\{0\} \times \mathbb{R}$; denote by $D(\tau)$ the subdisk of $h^{(0)}(\operatorname{tail}(\tau))$ bounded by the union of that segment and $\operatorname{tail}(\tau)$. Say that $\operatorname{tail}(\tau)$ is innermost if $\psi \cap \operatorname{Int} D(\tau)=\varnothing$.

4.1.4. Lemma. Let $\psi \subset S=S[i, j, \varepsilon]$ be a minimal braided $n$-star with $c(\psi)>0$. Then $(S, \psi)$ is isotopic to $\left(S^{\prime}, \psi^{\prime}\right)$, where $S^{\prime}=S\left[i^{\prime}, j^{\prime}, \varepsilon^{\prime}\right]$ is a braided surface with one more 0-handle and one more 1-handle than $S$, and $\psi^{\prime}$ is a minimal braided $n$-star with $c\left(\psi^{\prime}\right)<c(\psi)$. If $\varepsilon=+$ then we may take $\varepsilon^{\prime}=+$.

Proof. Since $c(\psi)>0$, there is a long ray $\tau \subset \psi$. Let $\tau$ be such that tail $(\tau)$ is innermost. Let $h^{(0)}(\operatorname{tail}(\tau))=: h_{x_{0}}^{(0)}, h^{(1)}(\operatorname{coccyx}(\tau))=: h_{z_{0}}^{(1)}$. There are eight cases, according as $x_{0}$ is $i\left(z_{0}\right)$ or $j\left(z_{0}\right), \varepsilon\left(z_{0}\right)$ is + or - , and $\operatorname{pr}_{3}(\operatorname{tip}(\tau))$ is larger or smaller than $z_{0}$. By a sequence of twirls, a case in which $x_{0}=j\left(z_{0}\right)$ may be reduced to the otherwise similar case in which $x_{0}=i\left(z_{0}\right)$; by considering Mir $S$, a case in which $\varepsilon\left(z_{0}\right)=-$ may be reduced to the otherwise similar case in which $\varepsilon\left(z_{0}\right)=+$. Thus we may assume that $\varepsilon\left(z_{0}\right)=+$ and $x_{0}=i\left(z_{0}\right)$. The proof when $\operatorname{pr}_{3}(\operatorname{tip}(\tau))-z_{0}$ is positive (resp., negative) is indicated in Figure 4 (resp., Figure 5).

Here is a detailed description of what is going on in Figure 4 (Figure 5 is similar but simpler). (A): A neighborhood of $D(\tau)$ on $h_{x_{0}}^{(0)}$ and a region on $h_{j\left(z_{0}\right)}^{(0)}$ are shown, joined by $h_{z_{0}}^{(1)}$. The innermost tail $(\tau)$ (the thin solid line) crosses $h_{z_{0}}^{(1)}$, as do, possibly, other arcs of $\psi$ (collectively the thin shaded line). By 2.3.4, $\tau$ is not loose, so $z_{0}<z_{1}:=\max \left\{\left[\operatorname{pr}_{3}(\operatorname{coccyx}(\tau)), \operatorname{pr}_{3}(\operatorname{tip}(\tau))\right] \cap\left(i^{-1}\left(x_{0}\right) \cup j^{-1}\left(x_{0}\right)\right)\right\}$; part of $h_{z_{1}}^{(1)}$ is shown, and $\left\{x_{0}\right\} \times\{0\} \times(Z \cap] z_{0}, z_{1}[)$ is indicated with heavy dots. (B): An inflation of sign + introduces $h_{z_{2}}^{(1)}$, where $z_{1}<z_{2}<\operatorname{pr}_{3}(\operatorname{tip}(\tau)), i^{\prime}\left(z_{2}\right)=x_{0}$, and $j^{\prime}\left(z_{2}\right)$ is the successor of $x_{0}$ in $X^{\prime}$. (C): A slide up moves $h_{z_{1}}^{(1)}$ past $h_{z_{2}}^{(1)}$; then, in order, each $\left.h_{z}^{(1)}, z \in Z \cap\right] z_{0}, z_{1}\left[\right.$, moves past $h_{z_{2}}^{(1)}$ by a slide up or slip up, as appropriate (these moves are unobstructed because $j^{\prime}\left(z_{2}\right)$ is the successor of $x_{0}$ in $\left.X^{\prime}\right)$. (D): $h_{z_{0}}^{(1)}$ slides up over $h_{z_{2}}^{(1)}$, changing $\psi$ by isotopy to $\psi_{1}$ with $c\left(\psi_{1}\right)=c(\psi)+2$. (E): By an isotopy of tail $\left(\tau_{1}\right), \psi_{1}$ is replaced by $\psi_{2}$ with $c\left(\psi_{1}\right)=c(\psi)+1$. (F): After a final slide up, $(S[i, j, \varepsilon], \psi)$ has been replaced by $\left(S\left[i^{\prime}, j^{\prime}, \varepsilon^{\prime}\right], \psi_{3}\right)$ where $c\left(\psi_{3}\right)=c(\psi)$ and $\psi_{3}$ is loose. A final isotopy (not illustrated) replaces $\psi_{3}$ by $\psi^{\prime}$ with $c\left(\psi^{\prime}\right)<c(\psi)$.

4.1.5. Corollary. If $\psi$ is an $n$-star on a Seifert surface $S$, then there is a braided Seifert surface $S^{\prime}=S[i, j, \varepsilon]$ and an isotopy carrying $(S, \psi)$ to $\left(S^{\prime}, \psi^{\prime}\right)$, where $\psi^{\prime} \subset$ $S^{\prime(0)}$ is a minimal braided $n$-star. If $S$ is quasipositive, then we may take $\varepsilon=+$.

4.2. Proposition. Every Stallings plumbing is realizable as a braided Stallings plumbing; if the plumbands are quasipositive then so is the plumbing.

Proof. Let $S_{1}$ and $S_{2}$ be Seifert surfaces, $\psi_{s} \subset S_{s}$ an $n_{s}$-star. By 4.1.5, we may assume that $S_{s}=S\left[i_{s}, j_{s}, \varepsilon_{s}\right]$ is braided (and, if $S_{s}$ is quasipositive, that $\varepsilon_{s}=+$ ). Up to twirls of $S_{1}$ and $S_{2}$, we may further assume that $\psi_{1} \subset h_{\min X_{1}}^{(0)}, \psi_{2} \subset h_{\max X_{2}}^{(0)}$. Finally, given $h$, up to turns of $S_{1}$ (or $S_{2}$ ) we may assume that there is an increasing injection $H: Z_{2} \rightarrow \mathbb{R} \backslash Z_{1}$ which determines the isotopy class of $h$ as in 3.2.

4.3. Theorem. A Stallings plumbing $S_{1} *_{h} S_{2}$ is quasipositive iff both $S_{1}$ and $S_{2}$ are quasipositive.

Proof. One direction follows from 3.5, the other from 4.2. 
QUASIPOSITIVE PLUMBING

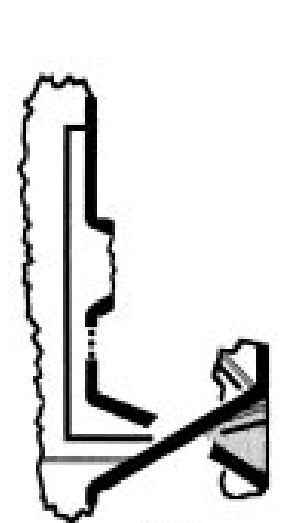

(A)

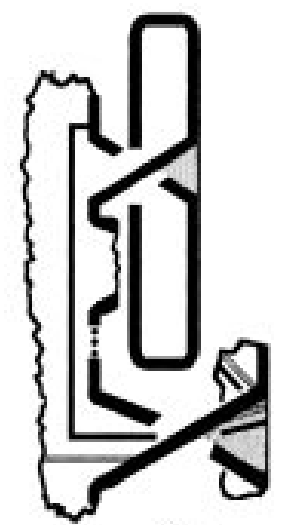

(B)

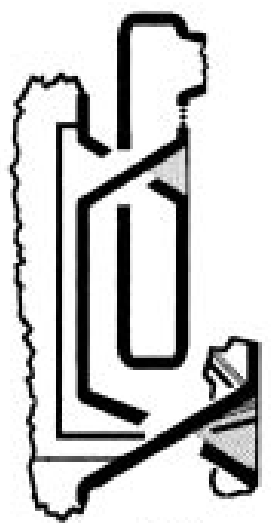

(C)

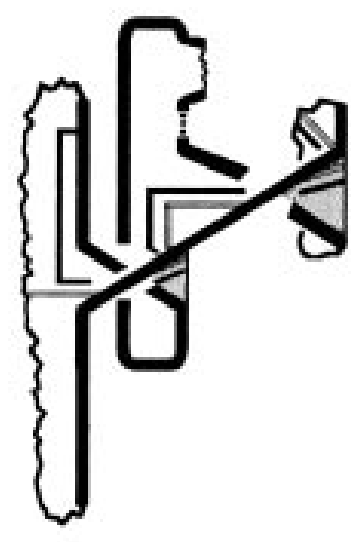

(D)

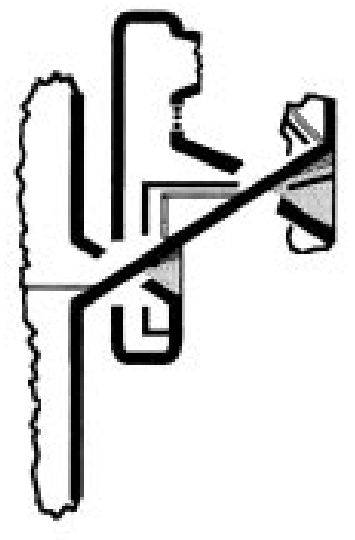

(E)

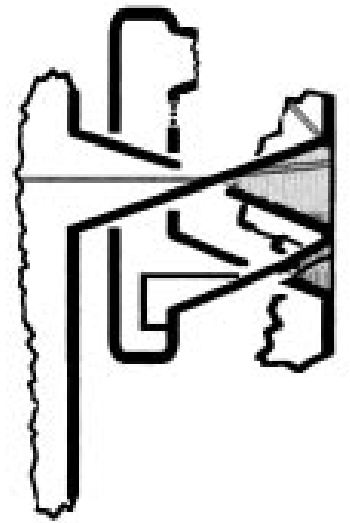

(F)

Figure 4

$\{3$

(A)

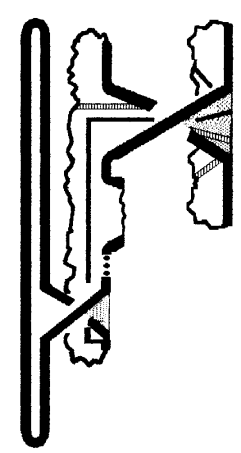

(B)

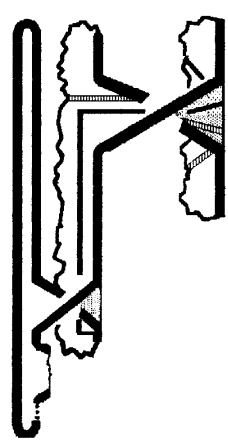

(C)



(D)

Figure 5 


\section{Applications}

5.1. Baskets. A Seifert surface

$$
S=\varepsilon_{\mu}\left(\left(\ldots \varepsilon_{2}\left(\left(D^{2} * A\left(O, n_{1}\right)\right) * A\left(O, n_{2}\right)\right) \ldots\right) * A\left(O, n_{\mu}\right)\right),
$$

where $A\left(O, n_{s}\right)$ is plumbed to $\left(\ldots\left(\left(D^{2} * A\left(O, n_{1}\right)\right) * A\left(O, n_{2}\right)\right) \ldots\right) * A\left(O, n_{s-1}\right)$ along $N_{s} \subset D^{2}, \varepsilon_{s} \in\{+,-\}$, and $N_{1}, \ldots, N_{\mu}$ are 2-patches such that $N_{i} \cap N_{j} \cap S^{1}=$ $\varnothing$ for $i \neq j$, will be called a basket, specifically a $\mu$-basket. (Note that, up to isotopy, the link $\partial S$ does not depend on the signs $\varepsilon_{s}$, but $S$ might.)

Examples. (1) Let $\Gamma$ be a planar tree, e $: V(\Gamma) \rightarrow 2 \mathbb{Z}$ an even weighting of its vertices, $P(\Gamma, \mathbf{e}) \subset S^{3}$ the corresponding plumbed surface [4], [16]. Induction on $\mu:=\operatorname{card}(V(\Gamma))$ shows that $P(\Gamma, \mathbf{e})$ is isotopic to a $\mu$-basket, [1], [15]. The link $\partial P(\Gamma, \mathbf{e})$ is arborescent in the sense of [4] and [16] (but not every arborescent link bounds a basket). By [4, 1.19], $P(\Gamma, \mathbf{e})$ is a fiber surface iff $\mathbf{e}(V(\Gamma)) \subset\{2,-2\}$ (but there are fibered arborescent links with fiber surfaces that are not baskets).

(2) If $\beta \in B_{n}$ is $\mathcal{T}$-homogeneous (where $\mathcal{T}$ is a suitable tree with $V(\mathcal{T})=\mathbf{n}$, cf. [15]), then the closed braid $\widehat{\beta}$ is fibered and its fiber surface is isotopic to a basket; the "homogeneous braids" in [17] are $\mathcal{J}_{n}$-homogeneous, where $\mathcal{J}_{n}$ has edges $\{k, k+1\}, 1 \leq k \leq n-1$. A fibered arborescent link $\partial P(\Gamma, \mathbf{e}), \mathbf{e}(V(\Gamma)) \subset\{2,-2\}$, is isotopic $([1],[15])$ to a closed $y_{\mu+1}$-homogeneous braid, where $y_{n}$ has edges $\{1, k\}$, $2 \leq k \leq n$.

(3) The link consisting of $N>0$ identically oriented fibers of the Hopf fibration $S^{3} \rightarrow S^{2}$ is a closed positive (hence $\mathcal{J}_{N}$-homogeneous) $N$-braid; its fiber surface (the Milnor fiber of $z^{N}+w^{N}$ at $\left.(0,0)\right)$ is, in effect, presented as a basket in [12].

5.1.2. Proposition. The $\mu$-basket in (5.1.1) is quasipositive iff $n_{s}<0$ for all $s$.

Proof. For $\mu=1$, this is proved in [13]. The result follows by induction, using 4.3 .

5.2. An inequality for the slice genus of a link. Let $L$ be a link of $r$ components. Let $\chi_{s}(L)$ be the greatest Euler characteristic $\chi(F)$ of a surface $F$ in $D^{4}$ with $L=\partial F$, so that the slice genus $g_{s}(L)$ equals $\left(2-r-\chi_{s}(L)\right) / 2$.

5.2.1. Lemma [14]. If $S$ is quasipositive, then $\chi_{s}(\partial S)=\chi(S)$.

5.2.2. Corollary. If $S$ is any Seifert surface, and $Q \subset S$ is quasipositive, then $\chi_{s}(\partial S) \leq 2 \chi(Q)-\chi(S)$.

Proof. Without loss of generality, $Q \subset \operatorname{Int} S$. Let $F \subset D^{4}$ be a surface with $\partial F=\partial S$ and $\chi(F)=\chi_{s}(\partial S)$. Let $Q^{\prime}:=(S \backslash \operatorname{Int} Q) \cup F$, so $\partial Q^{\prime}=\partial Q$. By 5.2.1, $\chi(Q) \geq \chi\left(Q^{\prime}\right)=\chi(S)-\chi(Q)+\chi(F)$, so $\chi_{s}(\partial S)=\chi(F) \leq 2 \chi(Q)-\chi(S)$.

Remark. For any given link $L$ presented as the closure $\widehat{\beta}$ of a braid $\beta \in B_{n}, 5.2 .2$. implies the slice-Bennequin inequality for $\beta$ [14]; and there are links $L$ (e.g., the fibered knot $6_{2}$, which is the closure of the homogeneous braid $\sigma_{1}^{3} \sigma_{2}^{-1} \sigma_{1} \sigma_{2}^{-1} \in B_{3}$ ) such that the bound on $\chi_{s}(L)$ given by some Seifert surface for $L$ is sharper than the slice-Bennequin inequality for any braid $\beta$ with $\widehat{\beta}=L$. In that sense 5.2.2 is stronger than the slice-Bennequin inequality. In another sense, they are equivalent, for (as observed in [14]) from the knowledge of the slice-Bennequin inequality for all braids, one can conclude 5.2.1, and thus 5.2.2, for all Seifert surfaces. 
5.3. The slice genus of the boundary of a basket. Let $S$ be a $\mu$-basket presented by (5.1.1). Let $p:=\operatorname{card}\left\{s: n_{s}<0\right\}$ and $q:=\operatorname{card}\left\{s: n_{s}>0\right\}$ (so $p+q \leq \mu)$; let $\partial S$ have $r$ components.

5.3.1. Proposition. $g_{s}(\partial S) \geq(1-r+|p-q|) / 2$.

Proof. The $p$-basket $S_{+}$with presentation derived from (5.1.1) by simply omitting plumbands $A\left(O, n_{s}\right)$ with $n_{s} \geq 0$ is quasipositive. By 5.2.2, $\chi_{s}(\partial S) \leq 2 \chi\left(S_{+}\right)-$ $\chi(S)=2(1-p)-(1-\mu) \leq 1-p+q$, so $g_{s}(\partial S) \geq(1-r+p-q) / 2$. By applying the argument to $\operatorname{Mir}(S)$, we also get $g_{s}(\partial S) \geq(1-r+q-p) / 2$. So $g_{s}(\partial S) \geq(1-r+|p-q|) / 2$.

\section{REFERENCES}

1. Michel Boileau and Lee Rudolph, Stein fillings via branched covers and plumbing, in preparation, 1995.

2. J. Conway, An enumeration of knots and links and some of their algebraic properties, Computational Problems in Abstract Algebra, Proc. Conf. Oxford, 1967, Pergamon Press, 1970, pp. 329-358. MR 41:2661

3. David Gabai, Foliations and the topology of 3-manifolds, J. Diff. Geom. 18 (1983), 445-503. MR 86a:57009

4. _ Genera of the arborescent links, Memoirs A.M.S. 339 (1986). MR 87h:57010

5. - The Murasugi sum is a natural geometric operation, Contemp. Math. 20 (1983), 131-144. MR 85d:57003

6. P. B. Kronheimer and T. S. Mrowka, Gauge theory for embedded surfaces. I, Topology 32 (1993), 773-826. MR 94k:57048

7. K. Murasugi, On a certain subgroup of the group of an alternating link, Amer. J. Math. 85 (1963), 544-550. MR 28:609

8. MR 30:1506

9. Lee Rudolph, Braided surfaces and Seifert ribbons for closed braids, Comment. Math. Helvetici 58 (1983), 1-37. MR 84j:57006

10. - Quasipositivity and new knot invariants, Rev. Mat. Univ. Complut. Madrid 2 (1989), 85-109. MR 90k:57009

11. , A congruence between link polynomials, Math. Proc. Camb. Phil. Soc. 107 (1990), 319-327. MR 90k:57010

12. _ A characterization of quasipositive Seifert surfaces (Constructions of quasipositive knots and links, III), Topology 31 (1992), 231-237. MR 93g:57014

13. _ Quasipositive annuli (Constructions of quasipositive knots and links, IV), J. Knot Theory Ramif. 1 (1993), 451-466. MR 94c:57017

14. $ـ$ Quasipositivity as an obstruction to sliceness, Bull. A.M.S. 29 (1993), 51-59. MR 94d:57028

15. __ Baskets, Hopf plumbing, $\mathcal{T}$-homogeneous braids, and arborescent surfaces, in preparation, 1997.

16. L. Siebenmann, Exercices sur les nouds rationnels, mimeographed notes, Orsay, 1975.

17. John R. Stallings, Constructions of fibred knots and links, Algebraic and Geometric Topology (R. James Milgram, ed.), Proc. Sympos. Pure Math., vol. XXXII, Part 2, Amer. Math. Soc., Providence, RI, 1978, pp. 55-60. MR 80e:57004

Department of Mathematics and Computer Science, Clark University, Worcester, Massachusetts 01610

E-mail address: lrudolph@black.clarku.edu 